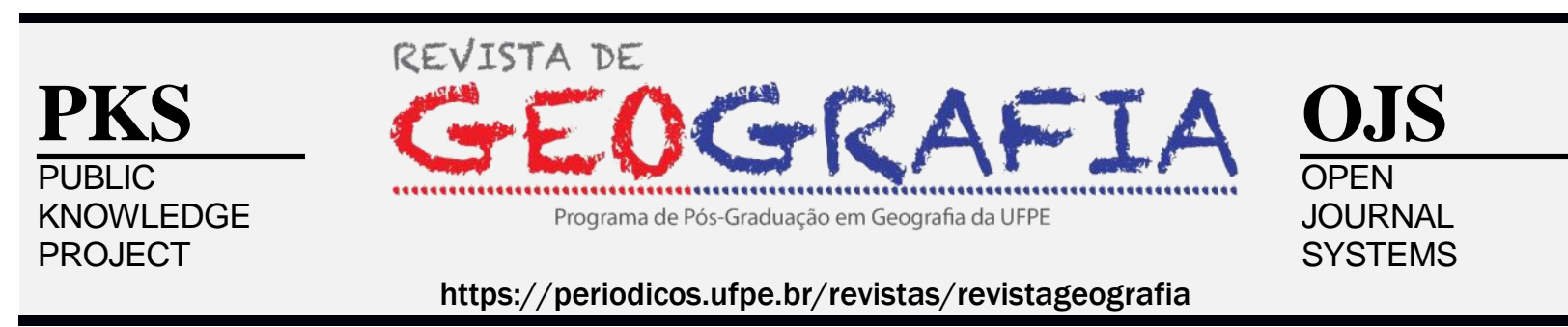

\title{
TEMPORALIDADES CIENTÍFICAS E TÉCNICAS NO BRASIL: UMA ANÁLISE ESPACIAL DO IMPACTO DA POLÍTICA NACIONAL DE CIÊNCIA, TECNOLOGIA E INOVAÇÃO EM SAÚDE E SEU FOMENTO À PESQUISA EM REDE
}

\author{
Allison Bezerra Oliveira ${ }^{1}$ \\ ${ }^{1}$ Universidade Estadual da Região Tocantina do Maranhão-UEMASUL.E-mail: allisonbzr@gmail.com
}

Artigo recebido em 11/07/2018 e aceito em 23/11/2019

\begin{abstract}
RESUMO
O presente trabalho tem por objetivo proporcionar reflexão acerca da importância da compreensão do espaço geográfico também como resultado de temporalidades científicas e técnicas, utilizando como base analítica a atuação da Política Nacional de Ciência, Tecnologia e Inovação em Saúde (PNCTIS) e seu fomento à pesquisa em rede. Nesse percurso, utilizou-se o estado de Pernambuco como recorte espacial referencial em relação ao Brasil. Utilizou-se a área de Farmácia como recorte de área da Saúde a ser analisada e o período de 2005 a 2010 como recorte temporal. Metodologicamente, foram selecionados dados referentes a editais, grupos de pesquisa, projetos aprovados no Brasil como base material para análise do impacto da referida política pública e consequente compreensão das temporalidades espaciais existentes no Brasil. Os dados apresentados sugerem que há pouca compreensão das temporalidades geográficas brasileiras na atuação de políticas públicas como a PNCTIS, o que contribui para a manutenção dos desníveis científicos e tecnológicos do país e consequente reprodução de velhas disparidades.
\end{abstract}

Palavras-chave: Espaço Geográfico, Política Nacional de Ciência, Tecnologia e Inovação em Saúde, pesquisa em rede.

\section{SCIENTIFIC AND TECHNICAL TEMPORALITIES IN BRAZIL: A SPACE ANALYSIS OF THE IMPACT OF THE NATIONAL POLICY ON SCIENCE, TECHNOLOGY AND INNOVATION IN HEALTH AND ITS DEVELOPMENT IN NETWORK RESEARCH}

\begin{abstract}
The present work aims to provide reflection on the importance of understanding geographic space also as a result of scientific and technical temporalities using as an analytical basis the performance of the National Policy on Science, Technology and Innovation in Health (PNCTIS) and its promotion to research in network. In this course, the state of Pernambuco was used as a reference spatial cut in relation to Brazil. The pharmacy area was used as a cut-off for the health area to be analyzed, in addition to the period from 2005 to 2010 as a temporal cut-off. Methodologically, we selected data related to public notices, research groups, projects approved in Brazil as a
\end{abstract}


material basis for analyzing the impact of this Public Policy and consequent understanding of the spatial temporalities in Brazil. The data presented suggest that there is little understanding of Brazilian geographic temporalities in the performance of Public Policies such as PNCTIS, which contributes to the maintenance of the scientific and technological differences in the country and the consequent reproduction of old disparities.

Key-words: Geographic Space, National Policy on Science, Technology and Innovation in Health, network research.

\section{INTRODUÇÃO}

A análise de políticas públicas se insere dentro do escopo de atuação da ciência geográfica, dentre outras questões, pelo fato de que tais políticas são resultado de realidades espaciais. Uma política pública é concebida como o conjunto de ações desencadeadas pelo Estado - no caso brasileiro, nas escalas federal, estadual e municipal - com vistas ao atendimento a determinados setores da sociedade civil. Em outras palavras, ela em si é um mecanismo criado para resolução dos mais diversos problemas, das mais diversas ordens.

Se o espaço geográfico é o reflexo da sociedade, pois resulta das multiplicidades produzidas pelas atividades humanas, devemos então compreender que ele constitui a base material tanto para a construção quanto para a atuação de uma política pública. Daí a inevitabilidade de refletir sobre a importância do espaço para a atuação eficaz de qualquer mecanismo dessa natureza, em qualquer área.

Historicamente, a saúde é um dos grandes gargalos no processo de desenvolvimento espacial brasileiro. Várias debilidades podem ser apontadas neste contexto: a distância entre a realidade e a construção de uma real equidade na saúde; a dependência brasileira das grandes corporações farmacêuticas; a cada vez maior mercantilização da saúde; e, principalmente, a capacidade de gerar conhecimento científico nacional para atender a demandas específicas do país.

Devido a essas e outras problemáticas no campo da saúde no Brasil, no ano de 2004 foi criada a Política Nacional de Ciência, Tecnologia e Inovação em Saúde (PNCTIS) com o objetivo de "contribuir para que o desenvolvimento nacional se faça de modo sustentável, e com apoio na produção de conhecimentos técnicos e científicos ajustados às necessidades econômicas, sociais, culturais e políticas do País". A PNCTIS assume assim dois aspectos importantes dentro de suas diretrizes: o primeiro deles é o de promover o estímulo ao desenvolvimento de conhecimentos científicos e tecnológicos em consonância com as necessidades nacionais, e o segundo deles está na adoção da pesquisa científica em rede como profícuo mecanismo para obtenção de resultados. 
Diante desse contexto, este trabalho tem por objetivo geral apresentar uma reflexão acerca da importância da compreensão do espaço geográfico também como resultado de temporalidades científicas e técnicas, utilizando como base analítica a atuação da Política Nacional de Ciência, Tecnologia e Inovação em Saúde (PNCTIS). Em outras palavras, abordaremos a importância do espaço geográfico como mecanismo fundamental para a compreensão de realidades e construção de qualquer política pública.

Dessa forma, para melhor delimitação da pesquisa, utiliza-se: a) Pernambuco como recorte espacial de referência, embora os dados analisados e coletados sejam de todos as Unidades Federativas do Brasil; b) os anos de 2005 a 2010 como recorte temporal, por ser período de implementação e maior ação da política em questão; c) a área de Farmácia como referência, dentre as áreas de saúde objetivadas pela política, por ser tratar de área intensiva na pesquisa científica e na produção e conhecimentos inseridos no Sistema Único de Saúde brasileiro; e d) o fomento à pesquisa científica em rede, entre as várias diretrizes da PNCTIS.

Considerou-se como hipótese que, uma compreensão não efetiva das diferenciações e temporalidades espaciais do país tende não só a dificultar a atuação de uma política pública dessa natureza, como também pode contribuir para a acentuação de problemas já existentes. Para comprovar a hipótese apresentada, analisou-se a forma de fomento promovida pela PNCTIS, e foram levantados dados referentes a projetos aprovados para a pesquisa científica em rede na área de Farmácia em Pernambuco e no Brasil, nos anos de 2005 a 2010, com o intuito de verificar eventuais desníveis existentes nacionalmente.

Dessa forma, o trabalho está organizado em quatro seções, além desta introdução e das considerações finais. Na primeira delas, "Uma breve revisão sobre o conceito de espaço na ciência geográfica", busca-se resgatar, mesmo que de forma breve, uma revisão da literatura acerca do conceito de espaço na ciência geográfica e da sua relevância para a compreensão do espaço como reflexo da sociedade. Em "A Política Nacional de Ciência, Tecnologia e Inovação em Saúde e o imperativo da pesquisa científica em rede" apresenta-se a PNCTIS, destacando seus objetivos, diretrizes e a introdução do fomento à pesquisa em rede em seu escopo de atuação.

Seguindo as seções anteriores, em "Multiplicidades espaciais: histórico da construção das instituições de ensino e pesquisa na área de Saúde em Pernambuco" faz-se um breve resgate histórico nordestino, em especial usando o estado de Pernambuco como exemplo, na construção de suas instituições de ensino e pesquisa e as debilidades provenientes desse contexto. E, por fim, em “O espaço geográfico enquanto temporalidades científicas e técnicas: 
os desníveis entre projetos aprovados para a pesquisa em rede na área de Farmácia em Pernambuco e no Brasil" são apresentados os desníveis no Brasil quanto aos projetos aprovados para a pesquisa em rede na área de Farmácia.

\section{O CONCEITO DE ESPAÇO NA CIÊNCIA GEOGRÁFICA: UMA REVISÃO NECESSÁRIA}

Para Lefebvre (1976), o espaço geográfico seria como a produção da sociedade, fruto da (re)produção das relações sociais de produção em sua totalidade. Essas relações se dariam, assim, em todos os âmbitos da vida cotidiana do homem. Corroborando com as ideias de Lefebvre (1976), Santos (2008), em seu livro Espaço e método, chama-nos a atenção para a reflexão de que sem produção - de qualquer natureza - é impossível existir o espaço.

Do espaço não se pode dizer que seja um produto como qualquer outro, um objeto ou uma soma de objetos, uma coisa ou uma coleção de coisas, uma mercadoria ou um conjunto de mercadorias. Não se pode dizer que seja simplesmente um instrumento, o mais importante de todos os instrumentos, o pressuposto de toda produção e de todo intercâmbio. Estaria essencialmente vinculado com a reprodução das relações (sociais) de produção. (Lefebvre, 1976, p. 34).

Assim o espaço é concebido como lócus de reprodução das relações sociais de produção, isto é, (re)produção da sociedade, quer sejam as relações entre homens e natureza, quer sejam os movimentos fabris de produção e transformação, ou ainda a construção de relações de socialização etc. O mérito do conceito "espaço geográfico" reside no fato de explicar teoricamente que uma sociedade só se torna concreta através do espaço que ela produz e, por outro lado, o espaço só seria inteligível através da sociedade (Corrêa, 1995; Moreira, 2012).

A palavra espaço, então, amplia seu significado em Geografia, abrindo para a disciplina extraordinárias possibilidades de explorações temáticas através de diversos espaços teóricos e relativos, possibilidades antes opacificadas pela concepção do espaço geográfico único. Como corolário, os objetos da Geografia também se multiplicam definidos pelos estudos das diversas relações entre os inumeráveis componentes do espaço geográfico. (Barros, 2003, p. 8).

Sendo assim, o espaço geográfico deve ser entendido como um conjunto, um processo, um sistema sempre contínuo e nunca fechado. Uma imbricação de trajetórias, sempre aberto ao 
inesperado, ao acaso, e que, como lócus da coexistência contemporânea, é marcado pela diversidade, diferença e multiplicidade (Massey, 2008).

O espaço não é um reflexo da sociedade, ele é a sociedade. [...] Portanto, as formas espaciais, pelo menos no nosso planeta, hão de ser produzidas, como o são todos os outros objetos, pela ação humana. Hão de expressar e executar os interesses da classe dominante, de acordo com um dado modo de produção e com um modo específico de desenvolvimento. (Soja, 1993, p. 89).

O espaço descrito por Lefebvre (1976), Soja (1993) e Moreira (2012) é aquele dotado de simultaneidade concreta e abstrata, a contextura das práticas sociais. É um espaço raramente visto, abstrato, e, diferentemente do que algumas abordagens no passado já haviam feito, ele não pode ser obscurecido por uma visão bifocal que o encare como apenas uma construção mental ou uma forma meramente física. Deve ser entendido também como relacional, sob múltiplos elementos.

O espaço é a dimensão implícita que molda nossas cosmologias estruturantes. Ele modula nossos entendimentos do mundo, nossas atitudes frente aos outros, nossa política. Afeta o modo como entendemos a globalização, como abordamos as cidades e desenvolvemos e praticamos um sentido de lugar. Se o tempo é a dimensão de mudança, então o espaço é a dimensão do social: da coexistência contemporânea de outros. (Massey, 2008, p. 15).

Portanto, tempo e espaço são realidades históricas que devem ser mutuamente consideradas como interdependentes sob uma perspectiva mais totalizadora, devem ser pensados também como elementos das transformações sociais. Logo, podemos buscar compreender as diferenciações temporais dos diversos espaços existentes no mundo e as consequentes temporalidades humanas daqueles que os produzem.

Se o tempo se revela como mudança, então o espaço se revela como interação. Neste sentido, o espaço é a dimensão social não no sentido da sociabilidade exclusivamente humana, mas no sentido do envolvimento dentro de uma multiplicidade. Trata-se da esfera da produção contínua e da reconfiguração da heterogeneidade, sob todas as suas formas - diversidade, subordinação, interesses conflitantes. (Massey, 2008, p. 97-98).

Afinal, a sociedade humana em processo é sempre um dos primeiros enfoques das análises geográficas e das ciências humanas no geral. Esse enfoque se dá sobre uma base material - o espaço e seu uso, o tempo e seu uso; a materialidade e suas diversas formas; as ações e suas diversas feições. Santos (2006) frisa a importância temporal na análise espacial 
dos fenômenos, destacando esse elemento como fundamental na composição estrutural do espaço.

Assim empiricizamos o tempo, tornando-o material, e desse modo o assimilamos ao espaço, que não existe sem a materialidade. A técnica entra aqui como um traço de união, historicamente e epistemologicamente. As técnicas, de um lado, dão-nos a possibilidade de empiricização do tempo e, de outro lado, a possibilidade de uma qualificação precisa da materialidade sobre a qual as sociedades humanas trabalham. (Santos, 2006, p. 33).

As técnicas são objetos explicativos do espaço, elas contêm aspectos empíricos da construção e evolução deste no tempo. O "aqui" deixa de ser tão somente uma delimitação física para tornar-se um espaço histórico (Freire, 1987). É por meio das técnicas que a evolução da lógica social humana e o espaço são construídos, afinal, o espaço geográfico é uma criação humana, portanto, é no meio natural, na terra que ele se revela.

O espaço geográfico é a base concreta da vivência terrena do homem. O ato de transformação consciente da natureza em meios de produção e de vida é um ato de construção consciente do espaço. E esse espaço é um fato que se revela na paisagem, em sua evolução de um ambiente dominado pela presença dos elementos primários da natureza (a primeira natureza) pelos de uma natureza progressivamente socializada pela ação transformadora do homem (segunda natureza). (Moreira, 2010, p. 103).

É por intermédio das técnicas que o homem no trabalho (em suas mais variadas formas) realiza a união entre espaço e tempo. Estas são periodizadas e incluem tempo qualitativa e quantitativamente. As técnicas são, portanto, uma medida do tempo: o tempo do processo direto de trabalho, da circulação, da inserção da ciência, da divisão territorial do trabalho e da cooperação.

Técnicas e tempo estão, com toda a evidência, fortemente entrelaçados. Ambas são outra coisa que não manifestações físicas ou biológicas de uma função material ou de um ritmo orgânico. Ambos estão fortemente mesclados à modelação de fenômenos e de processos sociais sempre novos. As relações entre a técnica e o tempo estão em geral muito mais emaranhadas do que as análises mais correntes, que as reduzem a relações de causa e efeito, nos querem fazer crer. (Horning, 1992, p. 49).

As técnicas participam na produção e na percepção do espaço e do tempo (tempo vivido, sensações de velocidade, diferenciações espaciais etc.). O espaço se destaca nas circunstâncias e condições que oferece para a produção, circulação, comunicação, cooperação e trabalho para o exercício do cotidiano da vida social como um meio operacional. Assim, técnicas são mecanismos operacionais, percebidos e constitutivos do espaço e do tempo. 
Se o espaço é o reflexo das sociedades e nele estão as diversas multiplicidades oriundas das ações humanas entre si e com a natureza, podemos considerar que este pode ser utilizado tanto como parâmetro metodológico para avaliar e compreender os diversos contextos, realidades e problemas, quanto para propor ações elucidativas, como no caso das políticas públicas, debatidas a seguir.

\section{A POLÍTICA NACIONAL DE CIÊNCIA, TECNOLOGIA E INOVAÇÃO EM SAÚDE E O IMPERATIVO DA PESQUISA CIENTÍFICA EM REDE}

A saúde historicamente é um dos grandes gargalos no processo de desenvolvimento espacial brasileiro. Ao longo da história, os esforços foram os mais diversos no intuito de melhorar os quadros e realidades nacionais. A promulgação da Constituição Federal de 1988, a qual estabelece o direito de todos à Saúde e a obrigação do Estado de provê-la, abriu novas e recentes portas para se pensar o papel do Estado enquanto agente principal das melhorias dos condicionantes de saúde. A criação de um Sistema Único de Saúde (SUS) foi, sem dúvidas, o avanço mais significativo nesse sentido.

O SUS pauta-se por três princípios constitucionais: universalidade, integralidade e equidade, todos frutos da Constituição de 1988. Do ponto de vista da Ciência \& Tecnologia, a aplicação desses princípios deve corresponder ao compromisso político e ético com a produção e apropriação de conhecimentos e tecnologias que contribuam para redução das desigualdades sociais em saúde. Um dos passos para reduzir esses gargalos é a produção de tecnologia para promoção de autossuficiência do atendimento no Sistema Único de Saúde.

Para garantir a autossuficiência do SUS é necessário, entre outros pontos, o fortalecimento da formação dos pesquisadores brasileiros e da produção de conhecimento científico e tecnológico em consonância com as necessidades do Estado, da sociedade e do mercado. A capacidade de colaborar para o desenvolvimento econômico e social coloca a pesquisa em saúde e o seu estímulo como estratégicos para a soberania do país.

Muitos foram os eventos, debates e ações políticas em prol da saúde até que somente em 2004 foi criada a primeira grande Política Nacional de Ciência, Tecnologia e Inovação em Saúde (PNCTIS) no Brasil. A “primeira grande" pelo fato de ser a primeira política desse porte desde a promulgação da CF de 1988, ademais, foi a primeira voltada para o desenvolvimento da saúde que considerou ciência, tecnologia e inovação em sua estrutura. O componente “inovação" só passou a ser efetivamente introduzido no ano de 1999 nas políticas nacionais. 
Assim, de acordo com a PNCTIS, a proposta é estimular o desenvolvimento de competências nacionais, com base nas necessidades do país. Formar mão de obra qualificada e estabelecer o dinamismo científico, tecnológico e inovativo na área de saúde; fixar os pesquisadores brasileiros no país; integrar os laboratórios e instituições de pesquisa; fortalecer a interação entre os setores privados, as universidades e os institutos de pesquisa; todos com vistas a promover a produção de conhecimentos em consonância com as necessidades nacionais. Desse modo, a PNCTIS foi criada com o objetivo de "contribuir para que o desenvolvimento nacional se faça de modo sustentável, e com apoio na produção de conhecimentos técnicos e científicos ajustados às necessidades econômicas, sociais, culturais e políticas do País" voltadas para a saúde (Brasil, 2008, p. 5).

O desafio, portanto, seria, ao mesmo tempo, fomentar o avanço do conhecimento científico na área da saúde e orientar os vetores de desenvolvimento tecnológico e de inovação da indústria de equipamentos, medicamentos, imunizantes e outros insumos básicos para a saúde, sempre sem perder de vista os mecanismos de apropriação societária dos resultados alcançados no conjunto de suas ações.

Uma PNCTIS voltada para as necessidades de saúde da população deveria ter como objetivo principal desenvolver e otimizar os processos de absorção de conhecimento científico e tecnológico pelas indústrias, pelos serviços de saúde e pela sociedade. O acatamento desta assertiva implica analisar o esforço nacional de C\&T em saúde como um componente setorial do sistema de inovação brasileiro. Por outro lado, essa perspectiva não deve sugerir uma visão reducionista ou utilitarista da política. Pelo contrário, reconhecendo a complexidade dos processos de produção de conhecimento científico e tecnológico neste setor, a PNCT\&I/S deve dar conta de todas as dimensões da cadeia do conhecimento envolvida na pesquisa em saúde. (Guimarães, 2004, p. 378).

Entre os vários mecanismos utilizados para o desenvolvimento de competências nacionais no campo da saúde, está a introdução do estímulo à pesquisa científica em rede como estratégia eficaz de obtenção de resultados mais expressivos e céleres. É a primeira vez que o estímulo à pesquisa em rede é introduzido em uma política desta natureza no Brasil.

Tal estratégia [da pesquisa em rede] visa a ampliar a capacidade de produzir conhecimentos para qualificar as decisões no âmbito da gestão pública. Desta forma, será possível suprir uma das maiores necessidades nas sociedades modernas, que é dispor de informações técnicas e científicas indispensáveis para fundamentar o processo de tomada de decisão, que têm forte impacto sobre diversos campos científicos e contribuem para o estabelecimento de um novo patamar nas relações entre ciência, Estado e sociedade (Brasil, 2004, p. $32)$. 
Dessa forma, converteu-se em um dos principais fios condutores da PNCTIS a publicação de fomento por meio da abertura de editais e fundos de financiamento para a construção de redes científicas dentro e fora do Brasil. A respeito de seu entendimento, estas podem ser descritas,

Como agrupamentos de pesquisadores e suas afiliações e grupos, em uma continuidade de pesquisa em rede, com vários níveis hierárquicos ou não, em espaços geográficos distintos ou não, onde se tem por finalidade a construção de laços de cooperação, confiança, reciprocidade e compartilhamento de experiências, promovendo, assim, o progresso da ciência por meio da produção de bens, serviços, conhecimentos e inovações. (Oliveira, 2014, p. 124).

Para Castells (1999, 2005), as redes se constituem na nova morfologia social da atual sociedade, e a difusão de sua lógica modifica de forma vital a operação de resultados dos processos produtivos, de experiência, poder e cultura. Assim, surge nos últimos anos uma significativa atenção para a abordagem das possibilidades de conexão humana e as consequentes perspectivas de trabalho coletivo, sobretudo na pesquisa científica.

Nesse sentido, as redes sociais podem ser compreendidas como formas independentes de coordenação de interações. A marca central da rede é a cooperação, baseada em confiança entre atores autônomos e interdependentes. Estes trabalham em conjunto por um período limitado de tempo e levam em consideração os interesses dos parceiros envolvidos, que estão conscientes de que essa forma de coordenação é o melhor caminho de alcançar seus objetivos particulares. É em função dessa capacidade de agregação que as redes têm um grande potencial para instigar processos de aprendizagem e são defendidas para a implementação de projetos de inovação, nos casos em que os riscos envolvidos apresentarem-se altos demais para cada um dos parceiros individualmente. (Frey, 2003, p. 175).

Diante desse contexto, a lógica instaurada foi a de que os pesquisadores brasileiros trabalhem em conjunto com pesquisadores de outras localidades dentro e fora do país. Essa concepção se expandiu para todas as demais políticas do segmento e agências de fomento como proposta metodológica de estimular a pesquisa científica, conforme nos apresenta o Conselho Nacional de Desenvolvimento Científico e Tecnológico (CNPq) em sua página:

O incentivo e apoio à formação de redes de pesquisa é extremamente eficaz para a indução ao desenvolvimento de uma dada área do conhecimento, o fortalecimento da capacidade instalada nas instituições de pesquisa e da realização de pesquisa colaborativa e interdisciplinar com integração entre as várias ciências, possibilitando a troca de metodologias, promoção do intercâmbio de dados, bem como a produção conjunta e a divulgação científica dos resultados. A articulação de redes científicas inter-regionais e 
interdisciplinares de pesquisa estimulará o intercâmbio entre instituições que concentram competências, a interação entre pesquisadores, o uso otimizado de recursos, o compartilhamento de infraestrutura para a pesquisa, com a perspectiva de convergência dos resultados.

Dentro do debate aqui já apresentado, é pertinente destacar algumas estratégias estabelecidas pela PNCTIS no Brasil. Para a sustentação e o fortalecimento do esforço nacional em Ciência, Tecnologia e Inovação em Saúde, uma das principais estratégias consiste em " $§$ 63. J) Esforços para criação de parcerias e redes de pesquisas nos países da América Latina, África e Ásia, visando a enfrentar problemas de saúde comuns" (Brasil, 2008, p. 22). Para a criação do Sistema Nacional de Inovação em Saúde:

$\S 65$. Requer a mobilização da totalidade da capacidade instalada de pesquisa, ensino, iniciativas de desenvolvimento tecnológico e inovação em saúde, numa perspectiva metodológica específica e intersetorial, incluindo redes de cooperação interinstitucional. [...]

$\S$ 66. [...] Dentre as ações, destacam-se a implementação de projetos de pesquisa e produção de conhecimentos cooperativos em rede e interinstitucionais. (Brasil, 2008, p. 22-23).

Para o setor da indústria farmacêutica, as estratégias propostas são: “a) Em curto prazo: implantação de uma rede nacional de informação de plantas medicinais; b) Em médio prazo: estímulo ao desenvolvimento de redes de cooperação técnico-científica; c) Em longo prazo: a produção de conhecimentos por meio de redes de cooperação que resultem em novos princípios ativos a serem inseridos no Sistema Único de Saúde” (Brasil, 2008, p. 24-26). Para a superação das desigualdades regionais: "§ 94 Estimular a criação de redes de cooperação" (Brasil, 2008, p. 29-30). E dentro do âmbito do Plano de Ação em Ciência, Tecnologia e Inovação (PACTI 2007-2010): "promover a formação de redes de pesquisa em todo território nacional".

O estímulo à construção de redes científicas é feito principalmente por meio das agências nacionais: Financiadora de Estudos e Projetos (FINEP), Coordenação de Aperfeiçoamento de Pessoal de Nível Superior (CAPES) e Conselho Nacional de Desenvolvimento Científico e Tecnológico (CNPq), que recebem verba por meio da PNCTIS e dos Ministérios da Saúde (MS) e de Ciência, Tecnologia e Inovação em Saúde (MCTIS).

São criadas várias modalidades que são direcionadas exclusivamente para a pesquisa em rede, ou mesmo agendas específicas de pesquisa, como o Plano Nacional de Ciência, Tecnologia e Inovação (PACTI), a Agência Nacional de Prioridades em Saúde (ANPS), ou editais lançados diretamente pelo Departamento de Ciência e Tecnologia (DECIT). 
No Brasil esse tipo de estímulo não existia nas agências de fomento antes da criação da PNCTIS. A incorporação da compreensão da pesquisa em rede se tornou tão expressiva nos últimos anos que mesmo o $\mathrm{CNPq}$ passou a considerar como mérito técnico-científico no julgamento de propostas, projetos que tivessem a possiblidade de criação de redes científicas entre pesquisadores. A Tabela 1, apresentada a seguir, mostra-nos que durante o período de 2006 a 2010 quase metade dos editais lançados pelo CNPq incluíram de alguma forma a dinâmica da pesquisa em rede em suas propostas, o que indica uma participação expressiva na totalidade dos editais.

Tabela 1: Participação de editais com fomento à pesquisa em rede no CNPq 2005-2010

\begin{tabular}{cccc}
\hline Ano & Pesquisas em rede & Pesquisas diversas & Total \\
\hline 2005 & 21 Editais & 32 Editais & $\mathbf{5 3}$ \\
\hline 2006 & 28 Editais & 30 Editais & $\mathbf{5 8}$ \\
\hline 2007 & 19 Editais & 26 Editais & $\mathbf{3 5}$ \\
\hline 2008 & 20 Editais & 44 Editais & $\mathbf{6 4}$ \\
\hline 2009 & 22 Editais & 39 Editais & $\mathbf{6 1}$ \\
\hline 2010 & 32 Editais & 38 Editais & $\mathbf{7 0}$ \\
\hline
\end{tabular}

Fonte: Organizado pelo autor a partir de dados do Diretório de dados do CNPq (2014)

A grande consideração nessa dinâmica é que o estímulo é feito quase totalmente em uma estrutura "horizontal e homogênea", em escala nacional ${ }^{1}$, com um único viés principal de fomento para todos os estados. Ou seja, os editais são lançados e todos os estados por meio de seus pesquisadores/instituições concorrem igualmente na submissão de projetos.

Se a proposta deste trabalho é refletir sobre o espaço geográfico também como resultado de temporalidades técnicas e científicas, e por considerarmos que o espaço brasileiro é formado por diversos outros espaços regionais, todos eles com diferentes temporalidades, levanta-se aqui a hipótese de que esse modelo de fomento tende a acentuar os já existentes desníveis tecnológicos no país. Antes, porém, de verificarmos a hipótese deste trabalho, abordaremos de forma breve a produção do espaço pernambucano à luz da construção das instituições de ensino e pesquisa.

\footnotetext{
${ }^{1} \mathrm{O}$ fomento pelas agências estaduais para a pesquisa em rede tem sido introduzido em suas diretrizes recentemente, contudo, em escala bem inferior. O fomento da PNCTIS é particularmente quase que em sua totalidade feito de forma nacional.
} 


\section{MULTIPLICIDADES ESPACIAIS: HISTÓRICO DA CONSTRUÇÃO DAS INSTITUIÇÕES DE ENSINO E PESQUISA NA ÁREA DE SAÚDE EM PERNAMBUCO}

Considerando que a técnica é elemento fundamental na produção do espaço e, por vezes, é a base para o avanço dos sistemas de objetos e sistemas de ações que possam vir a ser produzidos a seguir, considerando ainda que o espaço geográfico é dotado de multiplicidades, levando-nos à reflexão de que existem inúmeros elementos/dinâmicas que nos servem como base analítica para pensar o espaço, é perfeitamente factível aqui para nós a utilização das instituições de ensino e pesquisa como recorte analítico para o entendimento de contextos e temporalidades espaciais existentes no Brasil. O aqui e o agora são resultados não somente de hoje, mas principalmente do ontem.

Semelhante ao processo de ocupação do território brasileiro, desordenado e irregular, com regiões apresentando, ainda hoje, densidades populacionais maiores que outras, o processo de construção de instituições de ensino e pesquisa no Brasil se deu também de forma irregular e desigual. Para além da tardia criação destas no território nacional, quando falamos de Nordeste as disparidades são ainda maiores, como o caso de Pernambuco, que tratamos aqui neste artigo mais especificamente.

De acordo com Pimentel Neto (2008), o atraso na construção desse tipo de instituições em Pernambuco em relação a outras regiões como São Paulo, Rio de Janeiro e Minas Gerais gerou significativas disparidades espaciais ao longo dos anos. Somente em 1902 iniciou-se a construção da primeira Faculdade de Saúde em Pernambuco - justamente a Faculdade de Farmácia - localizada em Recife e impulsionada, sobretudo, pela Constituição de 1895.

Ou seja, somente no início do século XX tem-se a criação da primeira instituição de ensino superior do estado no campo da saúde. Somente uma década depois, em 1912, foi fundada a Escola Politécnica (atual Universidade de Pernambuco), que impulsionou posteriormente a criação da segunda faculdade do Recife, que foi a de Odontologia. E em 1920 foi criada a primeira Faculdade de Medicina do Recife.

Somente 22 anos depois é iniciada a criação do Instituto de Tecnologia de Pernambuco (ITEP) e, "é especialmente nesta onda que Pernambuco não consegue acompanhar o nível nacional, tendo um processo mais lento que os do Sudeste. No Brasil já estão surgindo desde 1932 as primeiras universidades" (Pimentel Neto, 2008, p. 57-58). O autor segue explicando esse processo mais detalhadamente: 
Porém devido ao atraso na criação das infraestruturas, é somente neste período [1940] quando está começando o processo de criação das universidades em Pernambuco. Antes disso, a única coisa que aparece de destaque é a criação do Sindicato de Médicos de Pernambuco (SIMEPE) e mesmo assim não é uma instituição de pesquisa ou/e ensino. Vale ressaltar que até esse momento, Pernambuco não apresenta iniciativas que incluam instituições de pesquisa científica. Desde o início da criação das instituições de ensino para a área de saúde a preocupação é a formação de profissionais na área. (Pimentel Neto, 2008, p. 58).

A primeira universidade do estado foi a Universidade Federal de Pernambuco, criada somente em 1946, catorze anos após o marco brasileiro de impulsionar a construção de instituições voltadas para o ensino e a pesquisa. E mesmo assim nela ainda não havia o curso de Medicina, que só foi incorporado no ano de 1958.

Em 1947, é criado o Centro Integrado de Saúde Amaury de Medeiros (CISAM) da UPE, com foco principal na formação de profissionais, ou seja, no ensino. Também é criada no mesmo ano a Fundação Joaquim Nabuco (FUNDAJ), que junto com o ITEP são as principais instituições de pesquisa deste período. E em 1949 é criada a Secretaria de Saúde de Pernambuco.

Em 1945 é criada a Faculdade de Ciências Médicas da Universidade de Pernambuco (UPE) e, em 1951 é criada a segunda universidade do estado, a UNICAP (Universidade Católica de Pernambuco). Já bem recentemente, em 1960, criou-se o Instituto Materno Infantil de Pernambuco (IMIP), hoje uma das principais instituições de referência em pesquisa médica do estado.

Nesse contexto, em 1964 é fundado o Laboratório Farmacêutico do Estado de Pernambuco (LAFEPE), principal referência em produção de medicamentos do estado, e somente em 1970 é criado um dos principais institutos de pesquisa de Pernambuco, o Centro de Pesquisas Aggeu Magalhães/Fiocruz (CPqAM), enquanto o Instituto Manguinhos/Fiocruz do Rio de Janeiro já havia sido criado em 1900, e o Centro de Pesquisa René Rachou/Fiocruz, em 1955 em Minas Gerais.

Em 1976, é fundado o Instituto de Ciências Biológicas - ICB, da Universidade de Pernambuco; em 1977 foi criada a Fundação de Hematologia e Hemoterapia de Pernambuco (HEMOPE); em 1979 é fundado o Hospital das Clínicas na UFPE; e em 1986, o Laboratório de Imunopatologia Keizo Asami (LIKA). Em toda essa trajetória de criação de instituições no estado de Pernambuco, cabe ressaltar que todas estavam concentradas na região metropolitana de Recife e em sua maioria eram voltadas apenas para o ensino, e não para a pesquisa. 
Isso não só concentrou na Região Metropolitana de Recife a esmagadora parcela dos pesquisadores ativos no estado, como também a construção de infraestrutura técnica, em especial o sistema de objetos necessário à realização da pesquisa (laboratórios, universidades, salas, equipamentos...). Somente no ano de 2000 criou-se o plano de interiorização das instituições de pesquisa, ensino e extensão no estado.

O contexto apresentado proporcionou, aliado a outras questões, um atraso de Pernambuco em relação a outros estados do país, sobretudo das regiões Sul e Sudeste, do ponto de vista da pesquisa científica. Os dados fornecidos pelo censo do CNPq (2014) dos grupos de pesquisa cadastrados na área de Farmácia, apresentados na Tabela 2, são didáticos quanto ao objetivo de apresentar as disparidades resultantes de contextos como o de Pernambuco.

Quando verificamos a quantidade de grupos de pesquisa cadastrados, linhas de pesquisa e pesquisadores envolvidos, os contrastes nacionais são grandes. Dos cinco estados com maior percentual de capacidade tecnológica envolvida com base nestes dados, são todos das regiões Sul e Sudeste. São Paulo, Paraná, Rio Grande do Sul, Rio de Janeiro e Minas Gerais ocupam respectivamente os primeiros lugares nos dados apresentados.

Tabela 2: Grupos de Pesquisa na área de Farmácia no Brasil em 2014

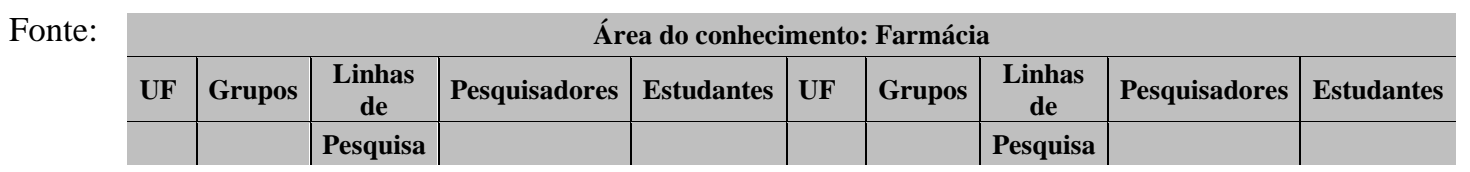

\begin{tabular}{llllllllll} 
SP & 97 & 357 & 624 & 662 & PA & 6 & 25 & 60 & 58 \\
PR & 43 & 188 & 316 & 377 & AL & 6 & 34 & 39 & 97 \\
\hline RS & 42 & 175 & 353 & 475 & RN & 5 & 37 & 135 & 227 \\
MG & 35 & 159 & 300 & 300 & AM & 5 & 20 & 40 & 58 \\
\hline RJ & 33 & 107 & 285 & 214 & ES & 3 & 5 & 22 & 27 \\
\hline SC & 27 & 121 & 174 & 299 & AP & 2 & 11 & 25 & 13 \\
\hline BA & 17 & 62 & 121 & 123 & MS & 2 & 9 & 11 & 28 \\
PE & 15 & 72 & 156 & 230 & PI & 2 & 5 & 19 & 33 \\
\hline PB & 13 & 79 & 132 & 163 & SE & 1 & 3 & 16 & 79 \\
CE & 13 & 75 & 123 & 184 & MA & 1 & 3 & 10 & 15 \\
\hline GO & 10 & 41 & 72 & 173 & MT & 1 & 5 & 6 & 3 \\
\hline DF & 6 & 53 & 48 & 77 & & & & &
\end{tabular}

Organizado pelo autor a partir de dados do diretório dos grupos de pesquisa do CNPq, Censo de 2014

As diferenças ficam maiores se observarmos que enquanto Pernambuco possui 15 grupos de pesquisa cadastrados, São Paulo possui 97 grupos. Também em comparação com Pernambuco, os estados do Paraná e Rio Grande do Sul apresentam quase o triplo de grupos, enquanto Minas Gerais e Rio de Janeiro aparecem com o dobro de grupos de pesquisa 
cadastrados. Estados como Maranhão, Sergipe e Mato Grosso apresentam apenas um grupo de pesquisa cadastrado com um volume muito pequeno de pesquisadores envolvidos. Já os estados do norte do país como Roraima, Rondônia, Amapá, Tocantins e Acre nem aparecem no censo respectivo.

Com relação ao volume de pesquisadores envolvidos, Pernambuco possui 156 membros cadastrados, enquanto São Paulo possui 624. Paraná, Rio Grande do Sul e Minas Gerais contam com aproximadamente o dobro de pesquisadores. Com instituições mais antigas e com maior capacidade de formação, o número de estudantes envolvidos tende a ser bem superior. Mato Grosso, por exemplo, possui três estudantes cadastrados, enquanto São Paulo possui 662, o que, por sua vez, é quase o triplo de Pernambuco.

Para além da simples comparação entre esses dados em nível nacional, uma vez que a análise destes pode ser considerada sob múltiplas vertentes, o que se pretende fazer compreender aqui é que os espaços brasileiros devem ser considerados sempre desiguais, pois o são de fato. E como tal, as políticas públicas devem ser construídas com maior nível de cautela na busca de potencializar as ações públicas e contribuir para a melhoria do quadro regional nacional, para não incorrerem como novos vetores de potencialização dos desníveis já existentes, como os apresentados a seguir.

\section{O ESPAÇO GEOGRÁFICO ENQUANTO TEMPORALIDADES CIENTÍFICAS E TÉCNICAS: OS DESNÍVEIS ENTRE PROJETOS APROVADOS PARA A PESQUISA EM REDE NA ÁREA DE FARMÁCIA NO BRASIL}

O espaço brasileiro é dotado de desigualdades, conforme já apresentado. E no campo da ciência isso não é diferente. Se associarmos essa reflexão a uma atuação vertical e homogênea em escala nacional para o estímulo à pesquisa por parte de uma política pública, que tipos de impactos teríamos? Dessa forma, levanta-se a seguinte questão: Qual o impacto da atuação da PNCTIS nos diversos estados brasileiros tomando como ponto de partida o fomento à pesquisa em rede?

Para comprovarmos nosso ponto de análise, tomando como ponto de partida exclusivamente a aprovação de editais para a pesquisa em rede, foram separados dados referentes a projetos aprovados (por estado) no Brasil, para essa modalidade, na área de Farmácia, entre os anos de 2005 e 2010, mediante a atuação da PNCTIS. A escolha da área se deu pelo fato de esta ter sido considerada como estratégica dentre todas as áreas da saúde e de 
o setor produtivo de fármacos e medicamentos ser considerado como estratégico pelas diretrizes da PNCTIS.

Os dados selecionados foram extraídos do Departamento de Ciência e Tecnologia do Ministério da Saúde e são referentes aos projetos aprovados em todas as agências de fomento brasileiras no período mencionado. Esses dados estão expressos na Tabela 3 a seguir.

Tabela 3: Projetos aprovados para a pesquisa em rede no Brasil 2005-2010

\begin{tabular}{|c|c|c|c|c|c|c|c|c|c|c|c|c|c|c|c|c|c|c|c|c|c|c|c|}
\hline \multirow[t]{2}{*}{ Ano } & \multicolumn{9}{|c|}{ Nordeste } & \multicolumn{4}{|c|}{ Sudeste } & \multicolumn{3}{|c|}{ Sul } & \multicolumn{3}{|c|}{ Norte } & \multicolumn{4}{|c|}{ Centro-Oeste } \\
\hline & $\mathrm{PE}$ & BA & $\mathrm{CE}$ & $\mathrm{RN}$ & PB & $\mathrm{AL}$ & MA & PI & SE & SP & RJ & MG & ES & RS & PR & SC & $\mathrm{AM}$ & PA & RO & DF & GO & MT & MS \\
\hline 2005 & 10 & 12 & 8 & 9 & & 2 & & 1 & 1 & 38 & 7 & 11 & 1 & 17 & 8 & 5 & & 6 & & 2 & 1 & & 1 \\
\hline 2006 & 10 & 10 & 11 & 5 & 3 & 2 & & & 1 & 23 & 60 & 17 & 3 & 9 & 4 & 1 & 1 & 1 & 1 & 2 & 3 & & \\
\hline 2007 & 4 & 1 & & & & 3 & & 1 & & 5 & 5 & 13 & & 2 & 2 & 1 & & & & & 1 & & \\
\hline 2008 & 3 & 4 & 3 & & 1 & & & & 1 & 14 & 19 & 12 & & 5 & 3 & & & 8 & & & 1 & 1 & 1 \\
\hline 2009 & 12 & 13 & & 4 & 6 & & 3 & 2 & 1 & 37 & 24 & 14 & 1 & 6 & 1 & 2 & 4 & 2 & & 6 & & 1 & \\
\hline 2010 & 4 & 6 & & & & 1 & 2 & & & 10 & 10 & 12 & & 1 & & & & & & & & & \\
\hline Total: & 43 & 46 & 22 & 18 & 10 & 8 & 5 & 4 & 4 & 127 & 125 & 79 & 5 & 40 & 18 & 9 & 5 & 17 & 1 & 10 & 6 & 2 & 2 \\
\hline
\end{tabular}

Os dados apresentados pela Tabela 3 mostram que nos estados das regiões Sul e Sudeste encontra-se maior acúmulo de projetos aprovados, e consequentemente maior captação de recursos, em relação às demais regiões. Enquanto São Paulo, Rio de Janeiro e Minas Gerais aprovaram 127, 125 e 79 projetos, respectivamente, estados como Mato Grosso do Sul e Mato Grosso aprovaram 2 projetos. Já Piauí e Sergipe aprovaram 4 projetos, e Rondônia apenas 1 no mesmo período, além dos estados que não aprovaram um único projeto sequer no período, como Amapá, Tocantins, Acre e Roraima. A região norte é a mais deficitária, com um total de 23 projetos aprovados em apenas três estados: Amazonas, Pará e Rondônia.

Os projetos aprovados dos estados das regiões Sul e Sudeste somam mais que o dobro dos estados do Norte, Nordeste e Centro-Oeste juntos. Resultados semelhantes são apresentados na Tabela 4, quando tratamos da quantidade de projetos aprovados pelo PACTI, no período de 2007 a 2010, para o setor de fármacos e medicamentos no Brasil na modalidade de pesquisa científica em rede. Nesse período, quase $50 \%$ de todos os projetos aprovados, além de quase $66 \%$ de toda a verba utilizada nacionalmente, foram para instituições localizadas no Sudeste. 
Tabela 04: Projetos aprovados para a pesquisa em rede no Brasil via PACTI ${ }^{2}$ (2007-2010)

\begin{tabular}{|l|r|r|r|r|r|r|}
\hline \multicolumn{7}{|c|}{ Setor de fármacos e medicamentos } \\
\hline \multicolumn{1}{|c|}{ PACTI (2007-2010) } & Centro-Oeste & Nordeste & Norte & Sudeste & Sul & Total \\
\hline Quantitativo de projetos aprovados & 20 & 24 & 21 & 103 & 39 & $\mathbf{2 0 7}$ \\
\hline Valor gasto (milhões) & 10,98 & 12,68 & 6,97 & 112,01 & 27,39 & $\mathbf{1 7 0 , 0 3}$ \\
\hline
\end{tabular}

Fonte: Organizado pelo autor a partir de dados da Finep e CNPq (2015)

Isso contribui para que as instituições dos espaços mais periféricos, com menor knowhow, pouco consigam agregar em termos de capacidade tecnológica ou possibilidade de desenvolvimento científico por meio do estímulo da PNCTIS, uma vez que a captação financeira é menor. A autonomia do controle do fluxo de informações traduzido na capacidade de escolher seus próprios vínculos e conexões para o estabelecimento de pesquisa e a maior democratização do conhecimento ficam comprometidos e tendem a se concentrar nos grandes centros.

Sob outro olhar, regiões mais pobres com maiores debilidades no campo sanitáriofarmacêutico, por exemplo, que necessitam de maiores investimentos para que gargalos e debilidades sejam sanadas são as que menos conseguem competir e captar recursos para que a busca pelo saneamento de vulnerabilidades sociais sejam sanadas.

Essas questões nos parecem mais gritantes quando transformamos os dados apresentados na Tabela 3 em mapas expressos na Figura 1, apresentada a seguir. Os mapas nos apresentam a imensidão de espaços opacos e espaços luminosos da pesquisa científica em rede na área de Farmácia brasileira. Outras disparidades espaciais acabam por ser reproduzidas também na pesquisa acadêmica. Os dados apresentam a evolução anual de captação de recursos por região, o que enfatiza as concepções apresentadas por Santos \& Silveira (2001, p. 264) sobre os espaços opacos e espaços luminosos existentes no Brasil,

Chamaremos de espaços luminosos aqueles que mais acumulam densidades técnicas e informacionais, ficando assim mais aptos a atrair atividades com maior conteúdo em capital, tecnologia e organização. Por oposição os subespaços onde tais características estão ausentes seriam os espaços opacos

De um lado os espaços luminosos sob o ponto de vista científico se concentra nos grandes centros da região Sul e Sudestes e portanto maior intensidade de cores e de outro, os espaços opacos representados expressivamente pela região Norte e boa parte dos estados das regiões centro-oeste e nordeste. $\mathrm{O}$ que nos leva a considerar obrigatoriamente que, se há acúmulo de

\footnotetext{
${ }^{2}$ Não há PACTI para os anos de 2005 e 2006.
} 
funções diretoras em certos lugares e a sua falta na maioria dos demais devemos admitir que existem espaços que comandam e espaços que obedecem sob o ponto de vista científico no Brasil.

Figura 1: Mapas de projetos aprovados para a pesquisa em rede no Brasil 2005-2010

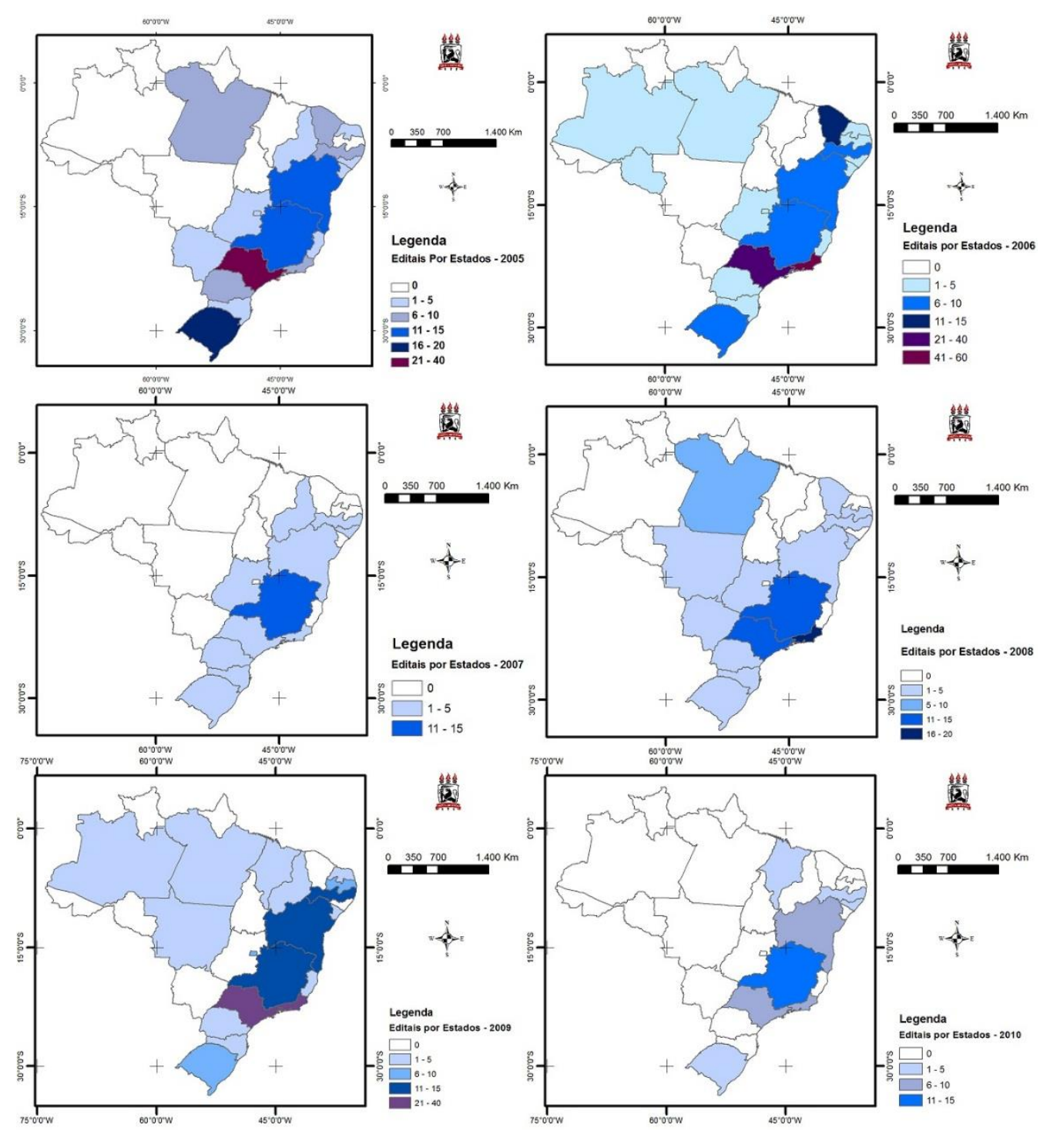

Fonte:

Organizado pelo autor a

partir de dados do Departamento de Ciência e Tecnologia - DECIT (2015)

Mesmo para os editais de encomenda, ou para editais específicos para determinadas regiões ou condicionantes de saúde local, a grande parcela das chamadas é de ordem nacional. Isso acarreta que, em algumas circunstâncias, instituições com maior número de pesquisadores e equipamentos de pesquisa aprovem um número maior de projetos em detrimento das 
instituições locais com menor capacidade "competitiva". Assim, regiões com maior densidade científica e técnica se sobrepõem àquelas com menos capacidade tecnológica.

Mais importante do que meramente expor disparidades em termos de aprovação de projetos e consequente destinação financeira, esses dados apresentam a problemática em torno da pouca sensibilidade e compreensão do espaço geográfico como mecanismo eficaz na avaliação de diferenciações e na proposição de mecanismos para a atuação das políticas públicas. Ademais, eles nos mostram que esta não compreensão não só contribui para resultados menos expressivos, como também para a acentuação de velhos problemas já existentes no Brasil.

Alguns estados e regiões pouco podem incorporar e controlar o direcionamento da construção de seus laços, uma vez que pouco lhe é permitido para tal. O fluxo acadêmico e as redes são concentrados nos grandes centros onde se determina as influências e hierarquias também para a pesquisa científica. A malha da rede científica brasileira acaba por reproduzir a malha anteriormente criada das redes de infraestrutura técnica, concentradas e controladas pelos grandes centros em detrimentos de outros.

Assim, centros menos desenvolvidos tecnologicamente ficam, em alguns casos, fortemente dependentes das conexões em rede estabelecidas pelos centros mais desenvolvidos, o que dificulta a emancipação tecnológica das regiões menos desenvolvidas cientificamente sobre sua capacidade de atração de fundos e pesquisadores, construção de infraestrutura, capacidade de desenvolvimento de tecnologias em consonância com suas realidades locais.

\section{CONSIDERAÇÕES FINAIS}

Mais do que fazer meras comparações, a proposta aqui levantada busca trazer ao seio do debate sobre o espaço geográfico, particularidades que possam ser utilizadas como base analítica na compreensão das diversas multiplicidades que acompanham a produção do espaço. Em específico, a interpretação dos espaços brasileiros à luz da produção técnica por meio das instituições de ensino e pesquisa.

Dessa forma, buscou-se, dentro do universo de componentes, mecanismos palpáveis enquanto orientação metodológica de visualização da importância do espaço na observação de diversos fenômenos. No caso da questão aqui apresentada, buscou-se a utilização de dados sobre o processo histórico de construção de instituições de ensino e pesquisa em Pernambuco, 
além dos dados sobre os grupos de pesquisa na área de Farmácia no Brasil e os resultados de editais aprovados.

Essas informações não só podem servir como base para a compreensão da importância do espaço geográfico na construção de políticas públicas, como para a avaliação da atuação destas. Assim, o entendimento sobre o espaço não só é fundamental, como deve ser feito sempre de forma cautelosa.

Dessa forma, os desníveis dos projetos aprovados no Brasil sugerem que um único viés horizontal de fomento não só tende a não conseguir introduzir de forma eficaz a dimensão dos espaços geográficos brasileiros, como proporciona a reprodução de velhas disparidades. Entre as quais podemos sugerir: a) concentração de maior capital humano articulado nos grandes centros; b) concentração da maior parte dos fluxos das redes em direção a alguns estados das regiões Sudeste e Sul; c) pouca capacidade de polarização científica e técnica em escala nacional; e) dificuldade de desenvolvimento científico e tecnológico em consonância com as necessidades locais das regiões mais periféricas do país.

Por fim, reiteramos o que apresentamos no início deste trabalho: o espaço geográfico deve ser sempre entendido como base material para a construção e também atuação de qualquer política pública. Essa compreensão deve ser pautada no entendimento de que o espaço é um produto histórico-social, com temporalidades e contextos distintos que se traduzem em singularidades e características próprias, que variam facilitando ou obstaculizando o desenvolvimento das mais diversas dinâmicas, como a pesquisa científica.

\section{REFERÊNCIAS BIBLIOGRÁFICAS}

BARROS, N. C. de. (2003) Ensaio sobre renovações recentes na geografia humana. Mercator, Fortaleza, v. 2, n. 4, p. 7-18.

BRASIL. (2004) $\mathbf{2}^{\mathbf{a}}$ Conferência Nacional de Ciência, Tecnologia e Inovação em Saúde. Brasília: Ministério da Saúde.

. Ministério da Saúde. Política Nacional de Ciência, Tecnologia, Inovação em Saúde

(PNCTIS). (2008) 2. ed. Brasília: Editora do Ministério da Saúde. In: http://bvsms.saude.gov.br/bvs/publicacoes/Politica_Portugues.pdf

CASTELLS, M. (2005) A Sociedade em Rede: do Conhecimento à Política. In: CASTELLS, M.; CARDOSO, G. (Org.). A sociedade em rede: do conhecimento à ação política. Imprensa Nacional de Portugal. p. 17-30

. (1999) A sociedade em rede - A era da informação: economia, sociedade e cultura. Vol. 1. 6. ed. São Paulo: Paz e Terra. 
CORRÊA, R. L. (1995) Espaço: um conceito chave na geografia. In: CASTRO, I. E. de.; GOMES, P. C. da C.; CORREAA, R. L. (Org.). Geografia: conceitos e temas. Rio de Janeiro: Bertrand Brasil.

FREIRE, G. (1987) Homens, engenharias e rumos sociais. Rio de Janeiro: Record.

FREY, K. (2003) Desenvolvimento sustentável local na sociedade em rede: o potencial das novas tecnologias de informação e comunicação. Rev. Sociol. Polít., Curitiba, n. 21, p. 165185.

GUIMARÃES. R. (2004) Bases para uma política nacional de ciência, tecnologia e inovação em saúde. Ciência \& Saúde Coletiva, Rio de Janeiro, v. 9, n. 2, p. 375-387.

HORNING, K. H. (1992) Le temps de la technique et le quotidien du temps. Paris.

LEFEBVRE, H. (1976) Espacio y política: El derecho a la ciudad, II. Barcelona: Ediciones Península.

MASSEY, D. (2008) Pelo espaço: uma nova política da espacialidade. Rio de Janeiro: Bertrand Brasil.

MOREIRA, R. (2012) Geografia e práxis: a presença do espaço na teoria e nas práticas geográficas. São Paulo: Contexto.

. (2010) O pensamento geográfico brasileiro. Vol. 3: as matrizes brasileiras. São Paulo: Contexto.

OLIVEIRA, A. B. (2014) A importância do espaço geográfico na construção e funcionamento de redes científicas na área de farmácia em Pernambuco fomentadas pela Política Nacional de Ciência, Tecnologia e Inovação em Saúde (PNCTIS). 346 f. Tese (Doutorado em Geografia) - Universidade Federal de Pernambuco, Recife.

PIMENTEL NETO, J. G. (2008) Desarticulação entre a base de C\&T e a oferta de serviços de atenção à saúde: A "imaturidade" do sistema setorial de inovação em saúde no estado de Pernambuco. 155 f. Dissertação (Mestrado em Geografia) - Universidade Federal de Pernambuco, Recife.

SANTOS, M. (2006) A natureza do espaço: técnica e tempo, razão e emoção. 4. ed. 2. reimpr. São Paulo: EDUSP. . (2008) Espaço e método. 5. ed. São Paulo: EDUSP.

SANTOS, M. \& SILVEIRA, M. L.O (2011) Brasil: Território e sociedade no início do século XXI. $15^{\text {a }}$ Edição. Rio de Janeiro: Record.

SOJA, E. W. (1993) Geografias pós-modernas: a reafirmação do espaço na teoria social crítica. Rio de Janeiro: Zahar. 